\title{
Controle Restrito de Estímulos em Participantes com Síndrome de Down e Crianças com Desenvolvimento Típico
}

\author{
Camila Domeniconi \\ Aline Roberta Aceituno da Costa \\ Júlio César de Rose \\ Deisy das Graças de Souza \\ Universidade Federal de São Carlos
}

\begin{abstract}
RESUMO
Participantes com atraso no desenvolvimento podem responder sob controle de parte de estímulos compostos. Um método para investigar controle restrito de estímulos seria realizar treinos de discriminação condicional combinando estímulos simples e compostos e testar as respostas dos participantes aos elementos individuais dos estímulos compostos. O presente estudo realizou tarefas desse tipo com participantes com Síndrome de Down e crianças com desenvolvimento típico. Foram ensinadas tarefas de emparelhamento com o modelo por identidade envolvendo estímulos abstratos. Em seguida, foram realizados testes em tarefas de emparelhamento com o modelo simultâneo e com atraso de zero ou dois segundos com quatro tipos de tentativas: 1. modelos e estímulos de comparação simples; 2. modelos simples e estímulos de comparação compostos; 3 . modelos compostos e estímulos de comparação simples; 4. modelos e estímulos de comparação compostos. Os participantes responderam com bastante acurácia em testes de emparelhamento com o modelo simultâneo. Nos testes com atraso na apresentação dos estímulos de comparação, os adultos com Síndrome de Down cometeram mais erros do que as crianças com desenvolvimento típico. Esses resultados confirmam e estendem os de estudos prévios que verificaram que indivíduos com atraso no desenvolvimento podem não responder sob o controle de todos os elementos de um estímulo, o que tem importantes implicações para o planejamento e o monitoramento do ensino para esta população.
\end{abstract}

Palavras-chave: controle restrito de estímulo; estímulos compostos; emparelhamento com o modelo; Síndrome de Down.

\section{ABSTRACT \\ Restricted Stimulus Control in Participants with Down's Syndrome and Typically Developing Children}

Developmentally delayed individuals may respond to certain parts of complex stimuli. One method to investigate restricted stimulus control involves conducting training of conditional discrimination combining simple and complex stimuli and test the responses of participants to individual elements of the complex stimuli. This study carried out such tasks with participants with Down's Syndrome and typically developing children. Initial training involved identifying matching tasks involving abstract stimuli. Then tests were carried out with four types of trials: 1 . simple sample and simple comparison stimuli; 2 . simple sample and complex comparison stimuli; 3 . complex sample and simple comparison stimuli; 4. complex sample and complex comparison stimuli. Participants responded with considerable accuracy in simultaneous matching to sample tasks. In delayed matching to sample tasks, adults with Down's Syndrome showed more errors than typically developing children. These results confirmed and extended findings from previous studies in the literature indicating that individuals with a developmental delay may not respond under the control of all elements of a stimulus. The findings have important implications for designing and monitoring teaching procedures in this population.

Keywords: restricted stimulus control; complex stimuli; matching to sample; Down's syndrome.

Skinner (1953) propôs, em sua análise sobre o comportamento operante, três elementos básicos que participam de uma contingência de reforçamento: os estímulos antecedentes, a resposta e a consequência.
Desta maneira, a ocorrência de reforço para uma determinada resposta não depende simplesmente da resposta, mas também da presença dos estímulos antecedentes associados com a produção daquele reforço. 
Sendo assim, a discriminação dos estímulos antecedentes que sinalizam a probabilidade de reforço é selecionada no ambiente natural e cultural de seres humanos e infra-humanos por estar associada com a maximização da probabilidade de obtenção de reforçamento. Não é difícil imaginar que determinadas respostas têm maior probabilidade de reforçamento dependendo da situação em que ocorram. Por exemplo, a probabilidade de obtenção de um aumento salarial poderá depender da situação em que o funcionário fala com o seu superior, ou seja, se a empresa está em boa situação, se o trabalho do funcionário tem sido valorizado, se o superior está em um bom dia, entre outras. Desta maneira, as chances de sucesso do funcionário (ou seja, obtenção do aumento salarial) serão bem maiores se ele for capaz de discriminar o máximo possível de elementos que compõem a situação antecedente à sua resposta (pedir ao superior pelo aumento) e agir sob controle de todos estes elementos, escolhendo o momento mais apropriado ao seu pedido.

As respostas diante de situações complexas nas quais a obtenção de determinado reforço, por meio de determinada resposta, depende da discriminação refinada de uma situação específica (ou seja, situações como as descritas acima, nas quais uma mesma resposta poderá produzir reforçamento se determinadas condições estiverem presentes na situação ambiental) foram chamadas experimentalmente de discriminação condicional. $\mathrm{O}$ estabelecimento de relações de controle condicional foi primeiramente abordado por Lashley (1938) e vêm sendo extensamente utilizado em estudos sobre comportamento complexo (Debert, Matos \& Andery, 2006).

Tipicamente, o ensino de discriminações condicionais tem sido realizado com o procedimento de emparelhamento com o modelo, utilizando elementos (estímulos) de três conjuntos hipotéticos $\mathrm{A}\left(\mathrm{A}_{1}, \mathrm{~A}_{2} \mathrm{e}\right.$ $\left.A_{3}\right)$, B $\left(B_{1}, B_{2}\right.$ e $\left.B_{3}\right)$ e $C\left(C_{1}, C_{2}\right.$ e $\left.C_{3}\right)$. No ensino das relações $A B$, em cada tentativa um estímulo do conjunto A é apresentado como modelo, e os estímulos do conjunto B são apresentados como estímulos de comparação. Nesse caso, os participantes devem selecionar o estímulo de comparação $\mathrm{B}_{1}$ diante do modelo $A_{1}$, e não os estímulos $B_{2}$ e $B_{3}$, apresentados simultaneamente com $\mathrm{A}_{1}$. A escolha de $\mathrm{B}_{1}$ produz reforço, enquanto as escolhas de $B_{2}$ ou $B_{3}$ não produzem consequências programadas ou são seguidas por consequências não reforçadoras convencionadas para erros. De maneira similar, o reforço é contingente à escolha de $\mathrm{B}_{2}$ (e rejeição de $\mathrm{B}_{1}$ e $\mathrm{B}_{3}$ ) diante de $\mathrm{A}_{2}$, e à escolha de $\mathrm{B}_{3}$ (e rejeição de $\mathrm{B}_{1}$ e $\mathrm{B}_{2}$ ), diante de $\mathrm{A}_{3}$. No ensino das discriminações condicionais entre os estímulos dos conjuntos $\mathrm{A}$ e $\mathrm{C}$, diante de um estímulo modelo $\mathrm{A}_{1}$, a resposta consequente será a escolha do estímulo $\mathrm{C}_{1}$ ao invés de $\mathrm{C}_{2}$ e $\mathrm{C}_{3}$; do mesmo modo, as escolhas de $C_{2}$ e $C_{3}$ diante de $A_{2}$ e $A_{3}$, respectivamente, também serão reforçadas. Treinos deste tipo estabelecem relações de controle condicional entre os estímulos dos conjuntos $\mathrm{A}, \mathrm{B}$ e $\mathrm{C}\left(\mathrm{A}_{1} \mathrm{~B}_{1}, \mathrm{~A}_{2} \mathrm{~B}_{2}, \mathrm{~A}_{3} \mathrm{~B}_{3} ; \mathrm{A}_{1} \mathrm{C}_{1}, \mathrm{~A}_{2} \mathrm{C}_{2}\right.$, $\mathrm{A}_{3} \mathrm{C}_{3}$ ).

Estudos sobre controle de estímulos em situações experimentais têm mostrado que algumas vezes organismos diferentes respondem sob controle de apenas alguns aspectos da situação antecedente, o que pode provocar respostas incompletas, não adaptadas ou com reduzida probabilidade de reforçamento (Allen \& Fuqua, 1985; Domeniconi, de Rose, \& Huziwara, 2007; Dube \& McIlvane, 1997; Litrownik, McInnis, Wetzel-Pritchard \& Filipelli, 1978; Lovaas, Schreibman, Koegel, \& Rehm, 1971; Walpole, Roscoe, \& Dube, 2007). Este tipo de ocorrência tem recebido o nome de controle restrito de estímulos ou superseletividade e tem sido frequentemente demonstrado em indivíduos com autismo ou atraso no desenvolvimento.

Litrownik e col. (1978) realizaram um estudo com vinte e uma crianças, sendo sete com desenvolvimento típico, sete autistas e sete com Síndrome de Down, treinadas em tarefas de emparelhamento com o modelo simultâneo por identidade com estímulos bastante simples, que variavam em uma única dimensão (posição): eram duas linhas de cor marrom apresentadas na posição vertical ou horizontal. O participante deveria escolher o estímulo comparação que fosse igual ao modelo. O participante deveria escolher o estímulo comparação que fosse igual ao modelo. Quando os participantes acertavam seis tentativas em um bloco de oito iniciavam-se os teste. Os testes eram realizados em quatro blocos com oito tentativas cada nas quais eram possíveis dezesseis combinações que variavam quatro dimensões dos estímulos: forma (quadrado ou triângulo), cor (vermelho ou azul), tamanho (grande ou pequeno) e número de estrelas (uma ou quatro). As mesmas instruções do treino foram mantidas (olhar o modelo e apontar o igual) sem o fornecimento de qualquer consequência diferencial programada. Os resultados obtidos por Litrownik e colaboradores apontaram que todos os participantes com autismo e desenvolvimento típico foram capazes de responder a todas as quatro dimensões dos estímulos, enquanto as crianças com Síndrome de Down 
tiveram os desempenhos mais baixos. Os erros foram interpretados pelos autores como indícios de controle restrito de estímulos. Das sete crianças com autismo e das sete com desenvolvimento típico, apenas uma de cada grupo respondeu a duas dimensões dos estímulos (e não a quatro). No grupo das crianças com Síndrome de Down, seis das sete evidenciaram controle por uma ou por nenhuma das dimensões dos estímulos e nenhuma criança apresentou controle por todas as quatro dimensões. Os autores afirmaram que não existiu diferença estatisticamente relevante se comparados os desempenhos das crianças com desenvolvimento típico e autistas, mas o desempenho dos participantes com Síndrome de Down foi significativamente inferior, apontando a importância de se investigar sobre déficits de atençao com essa população.

Stromer, Mcllvane, Dube e Mackay (1993) seguiram essa linha de investigação sobre controle de estímulos e afirmaram que o procedimento então mais utilizado para o estudo de ocorrências de controle restrito de estímulos envolve inicialmente o estabelecimento de controle discriminativo por um estímulo composto por vários elementos. Em testes subsequentes esses elementos são separados a fim de possibilitar a análise da resposta individual a cada um destes elementos (ou seja, avaliar o controle que cada elemento exerce sobre a resposta; eg., Reynolds, 1961). Os resultados têm mostrado sistematicamente que indivíduos com desenvolvimento típico tendem a responder sob controle de todos os elementos que fazem parte do estímulo composto, enquanto indivíduos com atraso no desenvolvimento respondem sob controle de apenas um dos elementos que compõem o estímulo.

Com base nessa tradição de pesquisa, Stromer e cols. (1993) realizaram uma sequência de seis experimentos dos quais participaram sete indivíduos com déficits de desenvolvimento. Todos os experimentos envolveram tarefas de emparelhamento com o modelo nas quais foram manipulados: a quantidade de elementos que compunham os estímulos modelos e os estímulos de comparação e o atraso na apresentação dos estímulos de comparação (apresentação simultânea, com atraso de zero ou com atraso de um segundo).

No primeiro experimento cinco participantes realizaram tarefas de emparelhamento com o modelo por identidade, com quatro tipos diferentes de tentativas, dispostos em arranjos de emparelhamento com o modelo simultâneo e com atraso de zero ou um segundo: $\mathrm{O}$ primeiro tipo de tentativa apresentava modelos e estímulos de comparação simples ("SSS"), nas quais um dos estímulos de comparação era idêntico ao modelo e o outro era diferente dele; o segundo tipo de tentativa era formado por modelos compostos e estímulos de comparação simples ("CSS"), com um dos estímulos de comparação idêntico a um dos elementos do modelo composto e o outro era diferente de qualquer dos dois elementos do modelo; um terceiro tipo de tentativas apresentava modelos simples e estímulos de comparação compostos ("SCC") com o modelo sendo idêntico a um dos elementos de um dos estímulos de comparação e o estímulo incorreto apresentava dois elementos diferentes do modelo; o último tipo de tentativas apresentava modelos e estímulos de comparação compostos ("CCC"), nas quais um dos estímulos de comparação continha os dois elementos idênticos ao modelo e o incorreto era completamente diferente do modelo (não compartilhava nenhum elemento em comum com este).

Os resultados deste estudo apontaram que um dos participantes (JO) apresentou altos escores de pareamento simultâneo em tentativas do tipo: SSS, CCC e SCC e escores substancialmente mais baixos nas tentativas CSS. Outros três participantes (DC, EM e MM) apresentaram acurácia uniformemente alta em todas as tentativas de pareamento simultâneo e escores em pareamento com atraso tipicamente altos em todos os tipos de tentativas com exceção das tentativas do tipo CSS. Um último participante (AP) apresentou alta acurácia em todas as tentativas de pareamento simultâneo e tentativas com atraso de 0 segundos, porém apresentou diferença na acurácia entre tentativas tipo CSS e outros tipos de tentativas quando empregado o atraso de um segundo. Exceto para JO, nenhum dos participantes apresentou flutuação na acurácia dos escores em tentativas CSS. No conjunto, os resultados sugeriram que as respostas apresentadas pelos participantes estavam sob controle de apenas um dos elementos dos estímulos, quando estes eram compostos, especialmente nas tentativas do tipo "CSS". Esta tendência a responder sob controle de aspectos restritos dos estímulos compostos foi observada tanto nos arranjos de emparelhamento simultâneo quanto com atraso de zero ou um segundo, havendo resultados inferiores nas tentativas do tipo "CSS" com a introdução dos atrasos.

Os outros cinco experimentos da sequência do estudo de Stromer e cols. (1993) foram realizados com os mesmos participantes e arranjos experimentais, mas com alterações nos tipos de tentativas, ou seja, alguns tipos de tentativas foram substituídos por 
outros a fim de ampliar as investigações sobre o responder condicional. Assim, quando o controle era exigido por estímulos compostos, por exemplo, em tentativas do tipo "SCC" e "CCC" estas foram alteradas para tentativas do tipo "CSC" e "CCS", respectivamente. Dessa forma, passavam a existir tentativas com o modelo composto e um estímulo de comparação simples idêntico a um dos elementos do modelo e outro estímulo de comparação composto, com nenhum dos elementos idênticos aos do modelo e tentativas com modelos compostos e um dos estímulos de comparação também composto, com ambos os elementos idênticos aos do modelo e o outro simples. Em todos os experimentos foram confirmadas as altas porcentagens de acertos em tarefas de emparelhamento com modelos simples ("SSS", "SCC", "SCS" e "SSC") e porcentagens menores em tarefas com modelos compostos ("CSS", "CSC" e "CCC"), sugerindo que os participantes responderam sob controle de um dos elementos do modelo composto, o que caracterizaria um tipo de responder sob controle restrito de estímulos, ou superseletivo.

Apesar da literatura apontar o responder sob controle restrito de estímulos como comum em indivíduos com atraso no desenvolvimento, são raros os estudos que, de fato, comparam o desempenho desses participantes com o de participantes com desenvolvimento típico. O presente estudo teve o objetivo de ampliar as investigações de Stromer e cols. (1993) no Experimento 1, com dois grupos de participantes: crianças com desenvolvimento típico e participantes adultos com Síndrome de Down. Buscou-se verificar possíveis semelhanças ou diferenças nos desempenhos e investigar indícios de controle restrito de estímulos em ambos os grupos, em função tanto do tipo de tentativa quanto do atraso na apresentação dos estímulos de comparação.

\section{MÉTODO}

\section{Participantes}

Participaram do estudo sete crianças com desenvolvimento típico (nomes fictícios: Bruna, Letícia, Nara, Jéssica, Lucas, Leonardo e Pedro) com idades entre 5 e 6 anos e idades equivalentes no Peabody Picture Vocabulary Test (Dunn \& Dunn, 1981). Participaram também três adultos com Síndrome de Down (Paulo, Roberta e Sueli). Os adultos tinham idades cronológicas entre 20 anos e seis meses e 26 anos e oito meses quando realizaram o experimento, suas idades no Peabody Picture Vocabulary Test variaram entre três anos e nove meses e cinco anos e sete meses.

\section{Local, equipamentos e materiais}

As sessões foram realizadas em uma sala do Laboratório de Estudos do Comportamento Humano da Universidade Federal de São Carlos, especialmente designada para esta finalidade, não sujeita a interrupção ou barulhos externos.

Foram utilizados um computador Macintosh e monitor sensível ao toque, fones de ouvido, uma câmera filmadora, e o software MTS (Dube, 1991). Além disso, foram utilizados materiais escolares e pequenos brinquedos.

\section{Procedimento geral}

As sessões eram realizadas em cinco dias consecutivos e cada sessão tinha uma duração média ou aproximada de vinte minutos.

Foram utilizados como estímulos apenas figuras abstratas do programa MTS apresentadas em branco e preto, na posição central e em dois dos quatro cantos da tela do computador.

Cada tentativa tinha início com a apresentação de um estímulo modelo na posição central da tela do computador. Durante o treino o estímulo modelo continha apenas uma figura abstrata. Após a emissão, pelo participante, de uma resposta de observação ao modelo (um toque ao estímulo), a tentativa tinha sequência pela apresentação simultânea de dois estímulos de comparação (no emparelhamento com o modelo simultâneo) ou pelo desaparecimento do estímulo modelo por zero ou dois segundos (no emparelhamento com atraso) e, finalmente a apresentação dos estímulos de comparação. Os estímulos de comparação eram apresentados em duas das quatro janelas, posicionadas nos cantos da tela do computador; as posições de apresentação dos estímulos correto e incorreto eram aleatórias. Durante as tentativas de treino, as respostas corretas eram seguidas pela apresentação de uma tela com estrelas coloridas de diferentes tamanhos e uma sequência ascendente de tons e as incorretas eram seguidas por uma tela escura. $\mathrm{O}$ estímulo modelo podia ser simples ou composto (contendo duas figuras abstratas posicionadas lado a lado), dependendo do tipo de tentativa. Nas tentativas de teste as respostas do participante não tinham consequências programadas. Ao final de cada sessão os participantes ganhavam uma ficha, que poderiam trocar por itens de papelaria ou por pequenos brinquedos, dispostos em um armário, dentro do laboratório. 


\section{Condições experimentais}

\section{Pré-treino de relações de identidade com figuras familiares}

O pré-treino consistiu em 60 tentativas de emparelhamento com o modelo simultâneo com figuras familiares. Foram utilizadas seis figuras: xícara, carro, sol, lua, boneca e árvore. Um estímulo modelo era apresentado no centro da tela do computador. Uma mensagem sonora, solicitando ao participante uma resposta de observação ao modelo (toque ao estímulo), era apresentada por meio de um computador. Esta mensagem era: "Toque a figura". Após a resposta de observação do participante, um estímulo idêntico ao modelo era apresentado. Era esperado que o participante tocasse o novo estímulo. A resposta do participante ao novo estímulo era seguida pela apresentação da consequência programada para acertos (descrita acima); caso o participante não emitisse nenhuma resposta em cinco segundos, a mensagem sonora era repetida. Depois de três tentativas com a apresentação de um estímulo de comparação e da mensagem sonora, outras três tentativas apresentavam um estímulo de comparação e nenhuma mensagem sonora. Todas as outras 54 tentativas apresentavam dois estímulos de comparação ao invés de um (um deles idêntico ao modelo - estímulo de comparação correto; o outro diferente do modelo). As consequências programadas para acerto e erro eram, respectivamente, a apresentação de uma tela com estrelas coloridas ou uma tela escura. O critério de aprendizagem para esta condição experimental era de $100 \%$ de acertos em uma sessão.

\section{Treino de relação de identidade com figuras abstratas}

Esta sessão de treino consistiu em 30 tentativas com utilização de figuras abstratas em branco e preto, no mesmo formato de emparelhamento com o modelo simultâneo descrito no pré-treino, mas sem nenhuma dica. Era apresentado um estímulo modelo no centro da tela do computador e após a resposta de observação ao modelo, eram apresentados dois estímulos de comparação (um deles idêntico ao modelo e o outro diferente). As respostas eram consequenciadas diferencialmente, com a apresentação da tela com as estrelas coloridas em caso de seleção correta e da tela escura em caso de seleção incorreta. O critério de aprendizagem nesta fase era de $100 \%$ de acertos em cada sessão.

\section{Testes}

Quando o critério de acertos na fase de ensino era atingido, tinha início uma fase de testes objetivando comparar o desempenho dos participantes em quatro diferentes tipos de tentativas, em dois arranjos de emparelhamento com o modelo: simultâneo ou com atraso (atrasos de zero ou de dois segundos).

Os quatro tipos de tentativas testadas foram adaptadas do Experimento 1 do estudo de Stromer e cols. (1993): 1- tentativas tipo "SSS", envolveram apresentação de um estímulo modelo simples e dois estímulos de comparação também simples, sendo um deles idêntico ao modelo e o outro diferente; 2- tentativas tipo "CSS", com um estímulo modelo composto (contendo dois elementos simples) e dois estímulos de comparação simples (um deles idêntico a um dos componentes do estímulo modelo e o outro diferente); 3- tentativas tipo "SCC", com estímulo modelo simples e dois estímulos de comparação compostos (um deles contendo um dos elementos do estímulo idêntico ao modelo e o outro contendo dois elementos diferentes do modelo); 4- tentativas tipo "CCC", que envolveram estímulos modelos compostos por dois elementos e estímulos de comparação também compostos, sendo que o estímulo correto era aquele que continha ambos os elementos idênticos aos do modelo e o incorreto continha ambos elementos diferentes do modelo. A Figura 1 ilustra algumas dessas tentativas.

Foram planejadas três sessões de testes, cada uma delas contendo 64 tentativas, 16 de cada tipo, ou seja, "SSS", "CSS" "SCC" e "CCC". Na primeira sessão de testes a apresentação de modelos e estímulos de comparação era simultânea, na segunda sessão as tarefas de emparelhamento eram programadas com atraso de zero segundo entre o término da apresentação do estímulo modelo e a apresentação dos estímulos de comparação e na terceira sessão as tarefas eram programadas com atraso de dois segundos. Não foram planejadas consequências para respostas corretas ou incorretas durante os testes e não havia critério de acertos. Ao final da sessão, todos os participantes recebiam uma ficha que podia ser trocada por um item de papelaria disposto em uma "lojinha". 


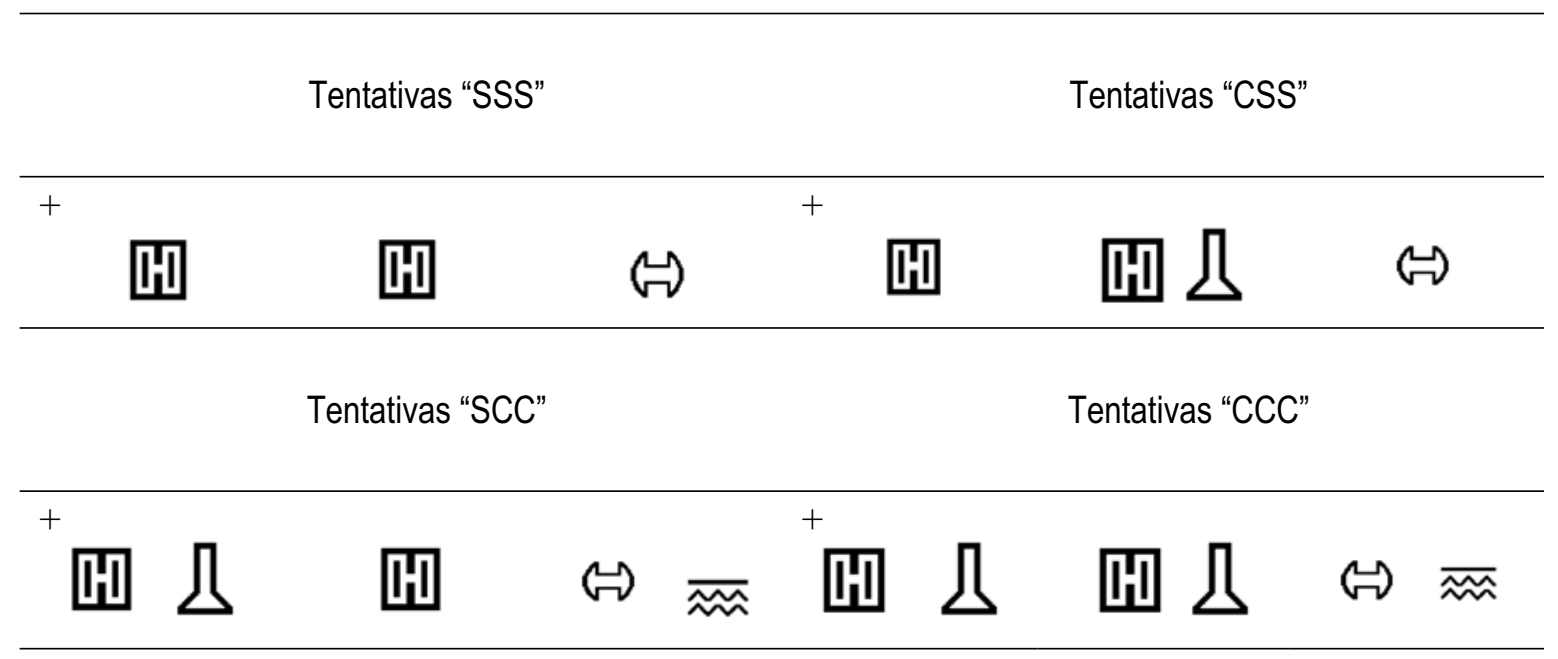

Figura 1. Exemplos dos quatro tipos de tentativas. No centro de cada tentativa estão os exemplos de estímulos modelo e nas laterais exemplos de estímulos de comparação. Os sinais positivos (+) indicam a escolha correta em cada caso (adaptada de Stromer e cols., 1993).

\subsection{Testes de emparelhamento com o modelo simultâneo}

Cada uma das 64 tentativas tinha início com a apresentação de um estímulo modelo (simples ou composto, a depender do tipo de tentativa empregado). Após a resposta de observação ao modelo esse estímulo permanecia disponível na tela do computador, dois estímulos de comparação (também simples ou compostos, a depender do tipo de tentativa em vigor) eram apresentados e o participante deveria tocar sobre um deles.

\subsection{Testes de emparelhamento com o modelo com atraso de zero segundo}

Cada tentativa tinha início com a apresentação de um estímulo modelo (simples ou composto, a depender do tipo de tentativa empregado); após a resposta de observação ao modelo, o mesmo desaparecia e, imediatamente, eram apresentados dois estímulos de comparação (também simples ou compostos, a depender do tipo de tentativa em vigor). O participante deveria tocar sobre um deles para o encerramento da tentativa.

\subsection{Testes de emparelhamento com o modelo com atraso de dois segundos}

As tentativas eram idênticas às descritas no item 3.2, com uma única diferença: após a resposta de observação ao modelo, o mesmo desaparecia e, transcorridos dois segundos, eram apresentados os dois estímulos de comparação.

\section{RESULTADOS}

1 - Pré-treino de relações de identidade com figuras familiares

Todos os participantes atingiram o critério de $100 \%$ de acertos nas tentativas do pré-treino em uma (Bruna, Letícia, Nara, Lucas, Leonardo, Pedro, Roberta e Sueli) ou, no máximo, em duas exposições à sessão experimental (Jéssica e Paulo).

2 - Treino de relação de identidade com figuras abstratas

Todos os participantes atingiram o critério de $100 \%$ de acertos em uma única sessão.

\section{3 - Testes}

A Figura 2 mostra as porcentagens individuais de acertos nas diferentes tentativas de testes obtidas pelas crianças com desenvolvimento típico (Bruna, Jéssica, Letícia, Pedro, Nara, Lucas e Leo) e pelos adultos com Síndrome de Down (Sueli, Paulo e Roberta). 
Sim. 0s 2s
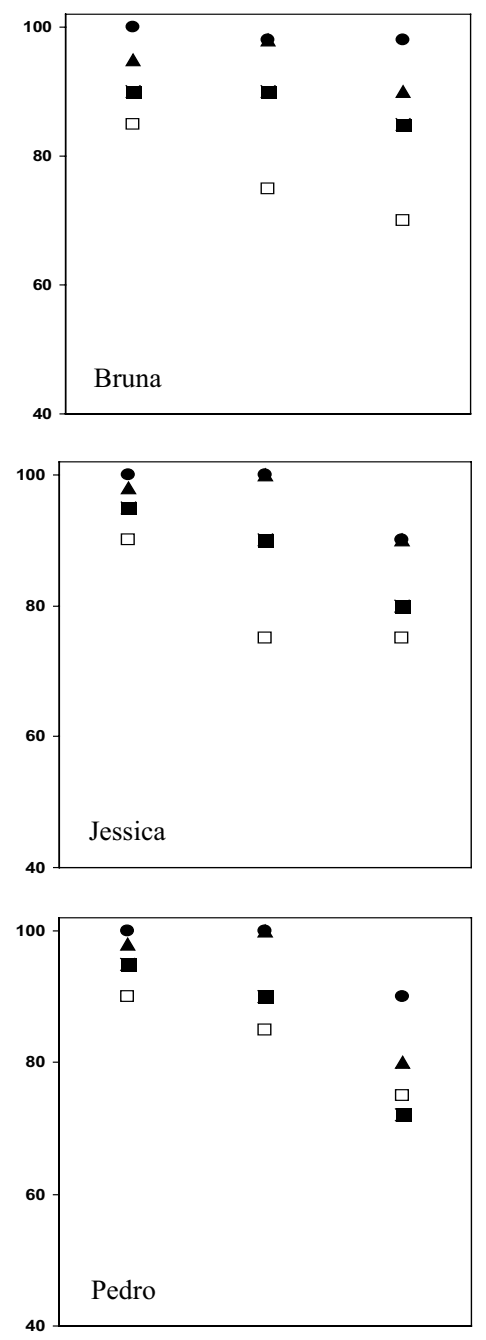

Sim. 0s 2s

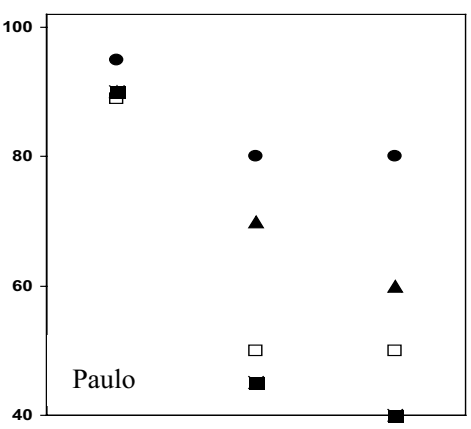

Sim. 0 s $2 s$
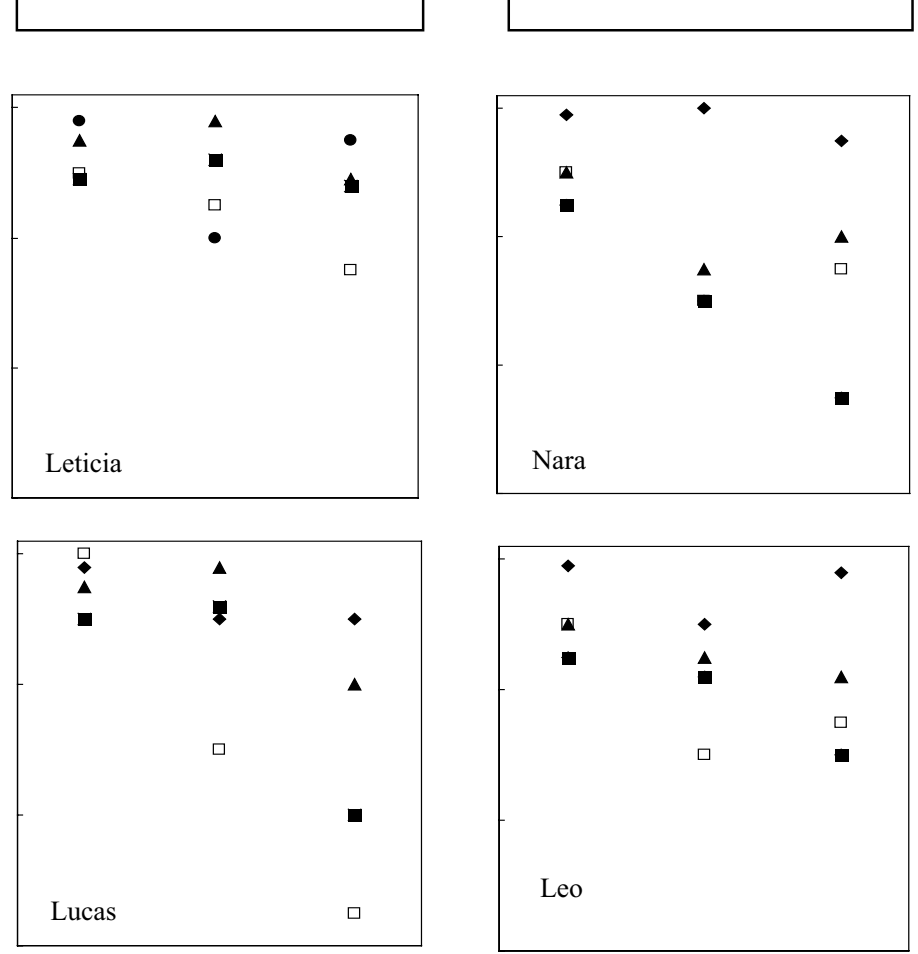

Sim. 0s 2s

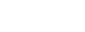


A primeira coluna de pontos de cada gráfico se refere às porcentagens de acertos nas tarefas de emparelhamento com o modelo simultâneo, a do meio com atraso de zero e a da direita representa os dados nas tarefas de emparelhamento com o modelo com atraso de dois segundos. Cada ponto mostra a porcentagem de acertos em um tipo de tentativa. Pode-se observar que, para todos os indivíduos, a porcentagem de acertos foi maior nas diferentes tentativas de emparelhamento com o modelo simultâneo, quando comparada com os emparelhamentos com atraso de zero ou dois segundos, especialmente nas tentativas dos tipos "SCC" e "CCC". Em geral, observa-se que todas as crianças, com exceção de Letícia na tarefa de emparelhamento com o modelo com zero segundo (cuja porcentagem de acerto mais baixa foi na tentativa "SSS"), obtiveram porcentagens de acertos mais baixas nas tarefas "SCC", "CCC" ou ambas. Quando o atraso na apresentação das comparações foi de zero segundo, Bruna, Nara, Pedro, Lucas e Leo obtiveram as porcentagens mais baixas nas tarefas "SCC", enquanto Jéssica, obteve desempenho mais baixo em ambas as tentativas "SCC" e "CCC". Quando o atraso aumentou para dois segundos, Bruna, Letícia, Pedro e Leo ainda mostraram maiores dificuldades nas tarefas "SCC", seguidas pelas "CCC"; ao contrário, Jéssica, Nara e Pedro tiveram mais dificuldades nas tarefas "CCC", seguidas pelas "SCC".

$\mathrm{Na}$ análise dos dados dos adultos com Síndrome de Down (Paulo, Sueli e Roberta), uma inspeção visual aos gráficos da Figura 2 permite dizer que a introdução do atraso na apresentação dos estímulos de comparação resultou em uma diferença, ainda mais acentuada do que a observada com as crianças, nas porcentagens de acertos nas diferentes tentativas, especial- mente nas compostas ("SCC", "CCC" e "SCS"). Quando foi introduzido o atraso zero, Sueli apresentou as porcentagens mais baixas nos três tipos de tentativas que envolviam estímulos compostos, "SCC", "CCC" e "SCS"; Paulo e Roberta, por sua vez, apresentaram as porcentagens mais baixas nas tentativas "SCC" e "CCC". Com o atraso de dois segundos, Sueli manteve as porcentagens mais baixas para os três tipos de tentativas compostas, Paulo também manteve o padrão de dificuldade encontrado quando o atraso era de zero segundo (ou seja, porcentagem de acertos mais baixa nas tentativas "CCC" seguidas de "SCC") e Roberta, que obteve as porcentagens mais baixas com atraso zero nas "SCC" e "CCC", com atraso de dois segundos, obteve porcentagens igualmente baixas nas "SCC", "CCC" e também "SCS", mostrando que o aumento do atraso dificultou ainda mais a tarefa para esta participante.

A Figura 3 mostra as médias de acertos para o grupo das crianças e dos adultos em cada um dos três tipos de tentativas. Nas tentativas de emparelhamento com o modelo simultâneo, as porcentagens de acertos variaram entre 85 e $100 \%$ para todos os participantes, sem diferenças notáveis nos desempenhos de participantes com desenvolvimento típico e com Síndrome de Down (porcentagens de acertos, de 93,5\%, em média para ambos os grupos, como pode ser observado na Figura 3). Em relação ao tipo de tentativa, apesar dos desempenhos nas tentativas do tipo "CSS" e "CCC" serem os mais baixos, quando comparado com os desempenhos nas tentativas "SSS" e "SCC", esta diferença também foi pouco acentuada (porcentagens de acerto, em média 90,5\% nas tentativas "CSS" e "CCC" e 96,5\% nas tentativas "SSS" e "SCC", para ambos os grupos, como demonstrado na Figura 3).

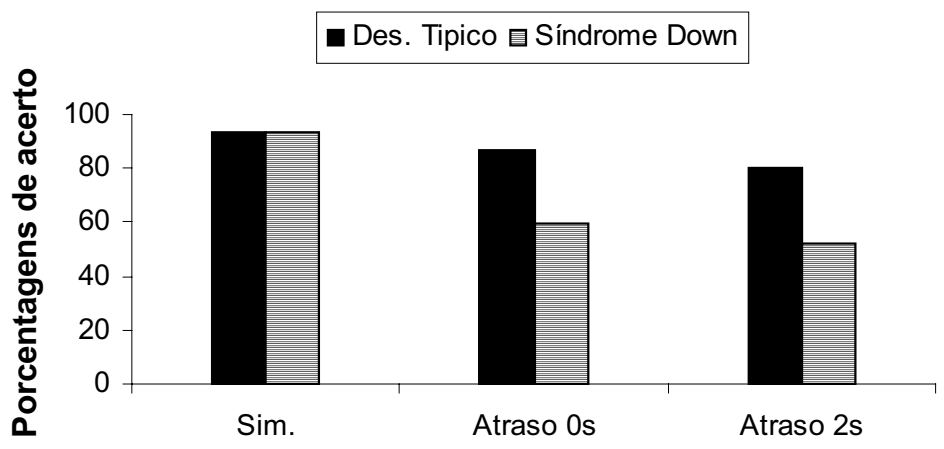

Figura 3. Médias de acertos dos participantes com desenvolvimento típico (barras pretas) e com Síndrome de Down (barras hachuradas) nos testes de emparelhamento com o modelo simultâneo, com atraso zero e com atraso de dois segundos. 
Os resultados das tentativas de emparelhamento com o modelo com atraso zero e de emparelhamento simultâneo mostraram diferenças expressivas de acordo com o exame visual dos desempenhos dos participantes com desenvolvimento típico em relação ao desempenho dos participantes com Síndrome de Down. As médias de acertos foram respectivamente, $86,6 \%$ e $59,5 \%$ (ver Figura 3 ).

A observação da Figura 3 permite comparar os desempenhos dos diferentes tipos de participantes nos emparelhamentos simultâneos e com atraso de zero segundo, pode-se observar que as médias foram, respectivamente, $93,5 \%$ e 73,5\%, assim, o atraso na apresentação dos estímulos de comparação foi uma variável importante no desempenho de ambos os grupos. Desse modo, em relação aos diferentes tipos de tentativas, as dificuldades apenas sinalizadas durante os emparelhamentos simultâneos foram acentuadas nos emparelhamentos com atraso, ou seja, as dificuldades foram maiores com tentativas do tipo "CSS" e "CCC", especialmente nessas últimas. Em média, os participantes com desenvolvimento típico obtiveram porcentagens de acertos de $92,5 \%$ nas tentativas do tipo "SSS" e "SCC" e médias de 80,7\% nas tentativas do tipo "CSS" e a única exceção nesta média foi a participante Letícia, que obteve $80 \%$ de acertos nas tentativas do tipo "SSS". Os participantes com Síndrome de Down, porém, obtiveram porcentagens de acertos de 71,3\% nas tentativas do tipo "SSS" e "SCC" e médias de 45,3\% nas tentativas do tipo "CSS" e "CCC". Nas tentativas de emparelhamento com o modelo com atraso de dois segundos pode ser observada ainda uma menor porcentagem de acertos quando comparada com as porcentagens de acertos nos emparelhamentos simultâneos. Assim, a porcentagem de acertos no emparelhamento com o modelo simultâneo foi em média $93,5 \%$ e no emparelhamento com atraso de dois segundos, 66\%. Quando comparados os desempenhos nas tentativas de emparelhamento com atraso de zero e dois segundos, a diferença é menos acentuada $(73,5$ e $66 \%$ de acertos, respectivamente).

Comparando as médias de acertos obtidos por participantes com desenvolvimento típico e com Síndrome de Down, durante os emparelhamentos com atraso de dois segundos observa-se que as porcentagens foram, respectivamente, 80,2 e $52,0 \%$.

Quanto aos tipos de tentativas, pode-se observar, nos desempenhos dos participantes com desenvolvimento típico, média de $86,2 \%$ de acerto nas tentativas do tipo "SSS" e "SCC" e média de 71,4\% de acerto nas tentativas do tipo "CSS" e "CCC". Os participantes com Síndrome de Down apresentaram, em média, porcentagens de acerto de 61,6 e $42,3 \%$, respectivamente.

\section{DISCUSSÃO}

O presente estudo utilizou procedimentos similares àqueles apresentados no primeiro experimento de Stromer e cols. (1993). O objetivo foi investigar os desempenhos de crianças com desenvolvimento típico e de adultos com Síndrome de Down, em tarefas de emparelhamento com o modelo simultâneo e com atraso de zero ou dois segundos, em tentativas com estímulos simples ou compostos.

Os dados replicaram aqueles obtidos por Stromer e colaboradores especialmente no que se refere ao desempenho dos participantes com Síndrome de Down (uma vez que no estudo original não participaram crianças com desenvolvimento típico). $\mathrm{Na}$ análise dos dados do estudo original pode-se observar que as porcentagens de acertos foram altas (em média 93\%) em todas as tarefas realizadas com emparelhamento com o modelo simultâneo. Esses resultados foram replicados no presente estudo com todos os participantes (crianças com desenvolvimento típico e adultos com Síndrome de Down). Em tentativas com estímulos compostos ou simples, foram registrados apenas alguns erros nas tentativas que apresentavam modelos compostos ("CCC" e "CSS").

Com a introdução do procedimento de emparelhamento com o modelo com atraso zero, as porcentagens de acertos dos participantes com Síndrome de Down diminuíram consideravelmente em relação às porcentagens de acertos obtidas durante as tentativas de emparelhamento com modelo simultâneo e esta diferença foi ainda mais acentuada nas tentativas cujos modelos eram compostos por dois elementos (médias de 45,3\% nas tentativas do tipo "CSS" e "CCC" comparativamente a médias de $71,3 \%$ nas tentativas do tipo "SSS" e "SCC"). Esta queda do desempenho dos participantes com Síndrome de Down pela introdução do atraso na apresentação dos estímulos de comparação foi confirmada com o aumento do atraso de zero para dois segundos, quando a porcentagem de acertos foi de $52 \%$ em média. Estes dados replicaram os dados obtidos no estudo original de Stromer e cols. (1993), sugerindo que os participantes com Síndrome de Down podem apresentar dificuldade em obter o máximo de reforçamento quando é exigido controle por estímulos com múltiplos elementos, apre- 
sentando, portanto, um tipo de responder sob controle restrito de estímulos.

O presente estudo obteve dados adicionais ao comparar os resultados obtidos por participantes com Síndrome de Down com resultados obtidos por crianças com desenvolvimento típico. Interessante observar que as crianças mostraram a mesma tendência observada nos resultados dos adultos. Foram observadas quedas no desempenho delas quando foi introduzido o emparelhamento com o modelo com atraso $(93 \%$ de acertos com emparelhamento simultâneo, $86,6 \%$ com atraso zero e $80,2 \%$ com dois segundos de atraso). Porém, o desempenho foi mais acurado quando comparado ao dos participantes com Síndrome de Down. Em relação aos tipos de tentativas, também parece que as dificuldades apresentadas pelas crianças foram mais acentuadas naquelas que exigiram controle por modelos compostos por dois elementos ("CSS" e "CCC") quando comparadas às tentativas com modelos simples ("SSS" e "SCC"), embora estas diferenças não tenham sido tão acentuadas como foi observado nos resultados dos participantes com Síndrome de Down.

Comparações dos desempenhos de crianças com desenvolvimento típico, autistas e indivíduos com Síndrome de Down, no que se refere ao controle de estímulos, também foram realizadas no trabalho de Litrownik e cols. (1978). Foram realizados treinos de emparelhamento com o modelo, utilizando modelos compostos, variando em quatro dimensões: cor, forma, tamanho e número de estrelas. Nos resultados, quando comparadas as médias de emparelhamentos corretos realizados por cada grupo de participantes nas quatro dimensões, os dados mostraram que crianças com desenvolvimento típico obtiveram percentuais próximos a $100 \%$ de acertos em todas dimensões. Os indivíduos com Síndrome de Down obtiveram índices significativamente menores de acertos, quando comparados aos outros dois grupos, evidenciando controle restrito de estímulos, segundo análises feitas pelos autores.

Apesar de muitos estudos apontarem os fenômenos característicos de controle restrito de estímulos como mais comuns em indivíduos com atraso no desenvolvimento intelectual ou com autismo, discute-se se estas ocorrências não poderiam resultar do tipo de estímulos empregados ou do arranjo experimental utilizado nos treinos. Allen e Fuqua (1985) afirmaram que, na literatura, existem muitas descrições de variabilidade nos resultados obtidos em treinos de discriminação condicional, inclusive em crianças com desenvolvimento típico. Algumas apresentam dificuldade em alcançar os critérios de aprendizagem com um tipo de arranjo experimental, mas conseguem quando este é mudado. Os autores discutem que a afirmação de que indivíduos com atraso no desenvolvimento respondem sob controle seletivo de estímulos pode ser muito pejorativa e causar a ideia de que o indivíduo é "incapaz" de adquirir o comportamento de discriminação de estímulos compostos, a despeito das diferentes estratégias e técnicas de ensino.

Independente da discussão sobre a natureza do responder sob controle restrito de estímulos, os resultados encontrados no presente estudo replicaram dados anteriores que mostraram ser mais comum este tipo de ocorrência em indivíduos com atraso no desenvolvimento do que em indivíduos com desenvolvimento típico, mas apontam também que esse padrão de responder não é inerente ao atraso (isto é, sob certas condições, participantes com desenvolvimento típico também podem responder sob controle parcial de estímulos). A produção deste tipo de conhecimento pode ser importante para o planejamento de procedimentos de ensino de diferentes repertórios para as mais variadas populações de aprendizes. Sabe-se, por exemplo, que na aprendizagem de leitura é fundamental que o responder do alfabetizando esteja sob controle de todas as unidades componentes da palavra, caso contrário, respostas incompletas ou erradas serão frequentes, podendo até levar à desistência do aluno.

\section{REFERÊNCIAS}

Allen, K. D., \& Fuqua, W. (1985). Eliminating selective stimulus control: A comparison of two procedures for teaching mentally retarded children to respond to compound stimuli. Journal of Experimental Child Psychology, 39, 55-71.

Debert, P., Matos, M. A., \& Andery, M. A. P. A. (2006). Discriminação condicional: Definições, procedimentos e dados recentes. Revista Brasileira de Análise do Comportamento, 2, 125-133.

Domeniconi, C., de Rose, J. C., \& Huziwara, E. M. (2007). Equivalência de estímulos em participantes com Síndrome de Down: Efeitos da utilização de palavras com diferenças múltiplas ou críticas e análise de controle restrito de estímulos. Revista Brasileira de Análise do Comportamento, 3, 47-63.

Dube, W. V. (1991). Computer software for stimulus control research with Macintosh computers. Experimental Analysis of Human Behavior Bulletin, 9, 28-30.

Dube, W. V., \& McIlvane, W. J. (1997). Reinforcer frequency and restricted stimulus control. Journal of Applied Behavior Analysis, 68, 303-316.

Dunn, L. M., \& Dunn, L. M. (1981). Peabody Picture Vocabulary Test-Revised. Circle Pines, MN: American Guidance Service.

Lashley, K. S. (1938). Conditional reactions in the rat. Journal of Psychology, 6, 311-324. 
Litrownick, A. J., McInnis, E. T., Wetzel-Prichard, A. M., \& Felipelli, D. L. (1978). Restricted stimulus control and inferred attentional deficits in autistic and retarded children. Journal of Abnormal Psychology, 87, 554-562.

Lovaas, O. I., Schreibman, L., Koegel, R., \& Rehm, R. (1971). Selective responding by autistic children to multiple sensory input. Journal of Abnormal Psychology, 77, 211-222.

Reynolds, G. S. (1961). Attention in the pigeon. Journal of the Experimental Analysis of Behavior, 4, 203-208.

Skinner, B. F. (1953). Science and human behavior. New York: Macmillan.
Stromer, R., McIlvane, W. J., Dube, W. V., \& Mackay, H. A. (1993). Assessing control by elements of complex stimuli in delayed matching to sample. Journal of the Experimental Analysis of Behavior, 59, 83-102.

Walpole, C. W., Roscoe, E. M., \& Dube, W. V. (2007). Use of a differential observing response to expand restricted stimulus control. Journal of Applied Behavior Analysis, 40, 702-712.

\section{Sobre os autores:}

Camila Domeniconi: Doutora. Universidade Federal de São Carlos.

Aline Roberta Aceituno da Costa: Doutora. Universidade Federal de São Carlos.

Júlio César de Rose: Doutor. Universidade Federal de São Carlos.

Deisy das Graças de Souza: Doutora. Universidade Federal de São Carlos.

Endereço para correspondência: Departamento de Psicologia - Laboratório de Estudos do Comportamento Humano - LECH - Rodovia Washington Luís, km 235 - SP-310 - 13565-905 São Carlos/SP - Endereço eletrônico: camila@ufscar.br 